Accelerator Division

Alternating Gradient Synchrotron Department

BROOKHAVEN NATIONAL LABORATORY

Upton, New York 11973

Accelerator Division

Technical Note

AGS/AD/Tech. Note No. 440

More BTA Lattice Matching

J. Niederer

August 12, 1996 


\title{
More BTA Lattice Matching
}

\author{
J. Niederer \\ AGS Department \\ Brookhaven National Laboratory
}

August 12, 1996

\section{Summary}

These notes critically examine the basic matching calculations that have guided the operation of the BTA transfer line. One of the paths followed has been to reduce dispersion at the point of Booster extraction, and thus be able to use the available quads for reducing amplitudes in the first part of the transfer line. This scheme can indeed produce compact, well behaved beams, but by itself is unable to satisfy AGS entrance conditions. Introducing additional quads along the BTA may be a practical way to obtain the needed conditions, along with some flexibility.

\section{Overview}

Over the years, various transfer line fitting programs have been routinely applied in hopes of properly matching Booster extraction conditions to AGS injection ones. For the most part, these adventures, when based upon the given BTA lattice and by now familiar conditions derived from lengthy AGS experience, have produced inconclusive or rather poor results. As proton beam intensities increase, beam losses limit intensities, and their radiation creates various nuisances if not outright problems. In practice, proton beam line tuning now tends to be based more on beam loss monitoring than upon reliable measurements and trusted models. As a sizable fraction of a people century has already been spent cranking routinely on models, these notes try to explore some less conventional approaches towards improving transport operations. They have tried to avoid further fine tuning, the engineering equivalent of polishing orange crates. Although there are basic mismatches among the Booster, the BTA, and the AGS, these studies have tried to find possible and significant improvements that are less traumatic than a complete rebuild of the BTA.

This particular matching problem has been festering for a number of years. Certainly a part of the problem is that something is seriously lacking among the matching programs themselves, the way that they are applied, and the way that information is presented to and from the programs. The original intent of these notes was to review these computing issues, and try to improve the programs and usage techniques accordingly, making it easier for more expert studies of the basic lattice design. The reviews indeed suggested improvements, easily done. The resulting changes and their displays pointed to likely lattice problems, which in turn could now be more readily analyzed, and so on, eventually leading to evaluations of some possible modest revisions of a few BTA 
lattice sections.

\section{Conventional Matching}

Booster extraction conditions are set mainly by the Booster lattice, and for the most part are not strongly tune dependent, except at the usual half integer and integer extremes. A reasonably stable set of values for the Booster ejection parameter sets of betas, alphas, and dispersion has been derived and checked to some extent over the years. A typical set of these values is given in the "Original Values" column of Table 1 . The Booster lattice is rather tightly focused. Extraction occurs at a point of maximum horizontal dispersion, which is not particularly helpful for tuning the BTA. Most applications take these Booster extraction parameters as given initial values for BTA tracking studies; adjusting these inputs is usually not considered in BTA work.

Simulated tracking through models of the BTA itself is generally subjected to several sets of constraints. The first of these is a general limit on the sizes of the beta functions, respecting aperture limits along the beam. A similar general limit on the dispersion amplitudes is often applied. At the end of the line, the $\mathrm{X}$ and $\mathrm{Y}$ phase ellipses are constrained, along with the $\mathrm{X}$ plane dispersion functions, to agree with preferred values for AGS acceptance. It is not at all clear what the margins of error are in these AGS acceptance conditions. As proton beams are spread all over the AGS aperture anyhow, maybe any such tolerances are irrelevant. Nevertheless, for our purposes here, typical lattice function values at the end of magnet QV15 and their assumed errors are given in Table 2. The common lattice cell matching programs treat the amplitude functions alpha and beta as independently constrained variables, even though they may be closely correlated. Similar correlations are very strong between the X and Px components of dispersion functions in the BTA lattice. For the BTA as built, these constraints amount to four sets of somewhat interdependent conditions which to date no one has managed to optimize together. The various BTA quad currents are treated as free parameters to be varied so as to satisfy the constraints. There are practical limits to these currents, which may be less than the nominal design limits in the range of $1000 \mathrm{amps}$, and which may also drag along optimization criteria.

The BTA constraints can also be weighted relative to each other, favoring particular constraints as being more important than others. Physically, strong focusing keeps the beam compact, reducing beam aperture losses in transport. This arrangement also affects the phase ellipses presented to the AGS, influencing AGS acceptance losses. While a number of such tradeoffs are present, relative weighting seldom seems to be used to explore preferences among the several sets of different constraints.

Usually these so called transfer line matches, if the computational mechanics are able to converge at all, are rather dependent upon the starting values assigned to BTA quads. Almost any starting combination will lead to some kind of "fit", seldom the same as with some other combination, with different juggling among the constraints. Naturally this behavior points towards a highly suspect landscape of the least squares minimizing function formed by the constraints and free parameters. There are rather deep, but narrow minima, scattered all over the place, superposed on a background many orders of magnitude above these minima. So far, no local minimum has been found to satisfy all of the imposed constraints at the same time. The approach to each such minimum can be adjusted in some way or another, but once in one of these holes, matching programs get 
stuck. For this kind of landscape, programs just drop into one of these minima near a set of starting values, and hence the dependence of fits on initial values of parameters. These sharp walls on landscape minima also imply very unstable BTA operating conditions, which of course are experienced. In some present configurations, changes of a few amperes of current in one or two BTA quads move a working BetaX from 50 to over 100, and such beams scrape chamber walls. While one can and does search out numerous starting patterns in hopes of chancing upon something better, this kind of landscape sends a strong message about the basic BTA design. It does not have the needed structure and adjustments for tuning independently the final $X$ phase ellipse, the $Y$ phase ellipse, and dispersion while maintaining aperture restraints. Because of the wavy nature of the BTA amplitude and dispersion functions, the amplitude constraints may be tripped a dozen or more times in a computed run through the transport line. In effect, each such episode ties up a magnet current parameter, so as a result, somewhat contrary to local folklore, the usual BTA matching has been attempted with more constraints than adjustment variables.

\section{Alternatives}

Given this evaluation, most of the choices for reducing transport losses, other than adding and rearranging BTA magnets, lie among adjusting BTA entry conditions in search of a more favorable fitting regime, and relaxing the AGS entry conditions. The practical effects of the entry options are to reduce the number of BTA adjustment parameters tied up in containing amplitudes. Hints about BTA beam sizes and characteristics and perhaps more stable operating configurations can be obtained from such paper studies. Effects on AGS capture and losses for a varied set of BTA exit parameters have not been simulated as yet by present orbit models. Perhaps one is merely moving BTA troubles from one place to another, or sharing the BTA ones with Booster extraction and AGS injection, but there do appear to be a number of areas that have yet to be explored.

A previous study by this writer has suggested that altering the BTA entry conditions, in particular reducing the dispersion, would lead to well behaved classes of matching solutions, ones that satisfied fully the stated AGS entry conditions. [1] These solutions are most sensitive to an initial Disp Px, expecting about .4, and are otherwise rather insensitive to small changes about some central set in the other initial parameters. These values are reproduced in the "BTA Fitted Values" column of Table 1. Accordingly, studies have been made to find practical ways to reduce dispersion in the beam delivered by the Booster. Generally, these trials try to increase focusing near the Booster F6 ejection region, without distorting the beam size too badly. With lower initial dispersion, in principle the strengths of the quad magnets in the front part of the BTA can be tuned to compact the beam, and to correct just the modest dispersion from the BTA bending magnets. BTA magnets further down the line can then be varied without unduly affecting the final dispersion. The existence of these classes of clean solutions under the use of six additional free parameters in the fitting also reinforces the view that the usual matching setup is over constrained - it needs more than the present 15 quad current parameters.

In the category of eternally springing hope, despite the apparent character of the fitting landscape, the simplex fitting routines have been modified somewhat to explore the parameter space with a broad range of randomly generated starting values, and iterated with larger currents than commonly used in practice. Other modifications optionally restart fits in new regions after symptoms of being stuck in a not very 
promising minima are triggered. While none of these exercises has turned up a more favorable region of the landscape, in principle this kind of broader search has to be tried, as the program obviously does get stuck in these dubious local minima. The migrad searching technique does far worse; if it converges at all, it is near some minimum far less satisfactory than found by the considerably modified simplex now in use here. Ellipse based constraints are used for AGS conditions, with realistic weighting, but, as expected, their improvements are overshadowed by the problems of getting proper $X$ and $Y$ phase advances while maintaining beam size. Random noise applied to the sides of the simplex during reduction doesn't help when nearby minima are separated by steep walls. Perhaps the usual styles of applying the matching programs are suspect - a parameter space of 15 current adjustments may be too many to expect sensible results. An alternative is to break the problem into tuning the first part of the line for small amplitudes and dispersion, and adjust the middle and / or end parts to achieve the preferred phase ellipses, relaxing amplitudes. These and many other variants have been tried, but so far the nature of the fitting landscape has persevered throughout these trials. Even the fitting routines themselves have been treated as suspect during these trials, but no mistakes have surfaced.

\section{Booster Studies}

A number of ways to modify the Booster outputs have been tried on paper, beginning with trials involving possible changes that might be easy to arrange and test in practice. These schemes have concentrated on modifying the dispersion, influenced by the already noted BTA study. Any incoming positive dispersion adds to the approximately $3 \mathrm{~m}$ generated by the two strong bends at the front of the BTA, compromising the momentum acceptance, and the local focusing pattern. Both $X$ and Px components of dispersion must be handled together, as they are strongly correlated here. Figure 1 shows undisturbed Booster dispersion in the $\mathrm{F}$ extraction region under typical operating conditions.

The relatively straightforward idea of splitting the Booster $\mathrm{X}$ and $\mathrm{Y}$ tunes produces little effect on dispersion until near an integer or half integer, however. Similarly disconnecting one or more quads is of little promise. Working around tunes of 3 and 4 is a little better, but most of the effect on dispersion also comes near half or full integers, and involves larger orbits, besides obvious problems with power supplies. Exchanging $\mathrm{H}$ and $\mathrm{V}$ quads produces a somewhat more attractive set of lattice functions with split tunes, but again, only near half and full integers, with larger amplitudes.

Among the schemes not so easy to rewire are various combinations of focusing changes among the Booster quad repertoire. Elaborate searches with parameter fitting techniques have failed to find a practical combination that would yield a zero or low $\mathrm{X}$ and a positive $\mathrm{Px}$ component of dispersion. On the other hand, a region of zero $\mathrm{X}$ dispersion at F6 can be readily obtained, accompanied by zero Px dispersion nearby. Increasing just the $\mathrm{F} 2$ quad strength by about $25 \%$ was as effective as any alternative tried. Dispersion resulting from one of the trials is shown on Figure 2. The curves are for F2 values of $1.25,1.27$, and 1.29 of the original value, for a typical match among tunes and dispersion just after Booster QF6. The corresponding increase in maximum BetaX and BetaY is from about 13 to the range of $25-30 \mathrm{~m}$. C. Gardner has worked on these approaches analytically, along the lines of the transition jump scheme for the AGS [2], 
guiding some of these searches which used tracking programs. The resulting $\mathrm{X}$ and $\mathrm{Px}$ dispersion components are quite sensitive to tunes, which might be helpful for adjusting the values delivered to the BTA around a nominal zero center, or conversely, may prejudice stability. An enhanced F2 setup has the obvious advantage of needing a single dedicated power supply if any of these ideas are actually to be tried. Among other possible schemes explored by parameter space searches, the phasing of the tightly focused Booster lattice works against sharing a dispersion perturbation effectively among other quads. Thus as a practical matter, the "easily achieved" Booster Disp X =0., Disp Px = 0 . solutions, along with their corresponding amplitude functions, were used as initial conditions to explore possible BTA transport setups. These representative initial conditions are shown as the "Booster Values" column of Table 1. Expected questions about whether the Booster can actually retain beam with such distortions are not addressed here.

\section{More BTA Studies}

The readily obtainable initial dispersion components and their corresponding amplitude functions are well outside of the range of fitted values indicated by the previous BTA matching runs of Set 2 of Table 1, with Disp Px appearing to be the critical item. Nevertheless, low entry dispersion should be expected to help with momentum acceptance through the BTA bends. Accordingly, the third set of entry conditions of Table 1 was tried with most of the variants discussed in the Section 3. above. The results were generally disappointing, with the same behaviors as for the original initial parameter set of Table 1. This series of runs added evidence that the basic matching procedures were severely faulted. The most obvious source of the problem is that there are not enough quads in the BTA both to compress the beam and to deliver the proper phase advances for AGS entrance conditions. If indeed BTA matching is underdetermined, adding quads should lead to better behaved solutions.

On paper, the easiest way to test this idea is to add a pair of quads in the space in the shielding wall, and repeat the calculations. (Case 2, new quads QH7A, QV7B) To keep things simple, the same kind of quad is used throughout these mythical changes. This match succeeds, satisfying all amplitude constraints of betas $<25 \mathrm{~m}$, dispersion $<2.6 \mathrm{~m}$, and lying within the margins of the given AGS entrance conditions. In addition, the sensitivity to all but the first two quads is such that the AGS entry margins are satisfied even if magnet currents are varied within a range of a few amperes. This result has something of the flavor that any amateur can toss in two magnets anywhere and get the BTA to work.

Unfortunately, when studied more carefully, it tends to look more like a lucky guess. The Case 2 configuration also fails to deal adequately with AGS entry dispersion when used with the original initial parameters of Table 1. A small initial dispersion appears to be necessary for it to work.

\section{BTA Magnet Shuffling}

Given these hints that there are at least some changes in the focusing patterns to which the BTA would respond favorably, some further exploratory studies have been carried out to find other kinds of changes that might be effective, at least in principle. The intent here is to find generic improvements that are worth the considerable effort that it may take to introduce them; these studies do not pretend to be either exhaustive or 
optimal. The positions of bends along the line have been retained to avoid survey complications for now.

The fits of Case 2 with two quads added in the wall region were overly sensitive to the first two quads in the line, and were a little touchy on the dispersion when the margins on the AGS conditions were reduced. The sensitivities suggested that the number of quads might still be marginal, and that adding more might be tried. Two additional quads were added in the Q10 - Q13 region, by reducing the larger remaining quad spacings and centering the $\mathrm{V}$ quads between pairs of $\mathrm{H}$ quads. The resulting lattice with two extra quads (Case 3: new QV10A and QH12B) produces nicely compressed beam amplitudes, but the early matching results were not especially better behaved. The reason is rather obvious - the focusing along this model of the line is now tight and regular, which leaves rather little play to adjust the $\mathrm{X}$ phase relative to the $\mathrm{Y}$ one at the AGS entrance. The simplex fits prefer to spike the Beta $X$ in the bend region to get this particular adjustment. As this spiking adversely affects the final dispersion, the tendency to spike can be manipulated with more severe amplitude constraints. The relative $\mathrm{X}$ and $\mathrm{Y}$ phase advances needed for the AGS can be tuned for matching purposes by constraining Beta $Y$ a little more strongly than Beta $X$. With these artifacts cleared up, this Case 3 configuration now works quite well.

As it might be expected that no one is about to rush out and rearrange the BTA shielding wall, and as the first impressions of the Case 3 configuration were not resoundingly better than that of Case 2, it has seemed useful to repeat Case 3 with the two mythical quads QH7A and QV7B turned off. (Case 4) Ideally, this also might help with understanding the somewhat delicate matter of numbers of constraints and free parameters. Narrow amplitude functions can still be maintained without the two wall quads. However, this Case 4 arrangement does not handle dispersion very well, matching behavior is inconclusive, and the setup does not respond very well to BTA tuning. When subjected to the original initial conditions of Table 1 , it has even worse problems with dispersion.

The sensitivity of the BTA line to quad currents in the first part of the line can be reduced somewhat further by several minor adjustments to the quad positions. Some fits are quite sensitive to the relative position of QV3 between $\mathrm{QH} 2$ and $\mathrm{QH} 4$; centering is preferred. Centering the QV5 quad between the DH2 and DH3 bends helps a little with dispersion. Otherwise, with zero initial dispersion, the front bending section can more or less operate undisturbed as a dispersion suppressor, with the usual strong focusing.

\section{Results}

Results for this set of studies are summarized in Table 3. A fit classed as "Very Good" satisfies all of the constraints, is stable against current drifts, and has a well defined penalty minimum about the same for all of the fitted variables. A "Bad" fit fails all of these criteria. The lone entry of "Maybe" has some problems with large dispersion and limited momentum acceptance, but is still better than the present arrangement, and doesn't involve Booster changes. Perhaps it can be beaten upon further. Cases for input set \#2 were not followed because there is no obvious way for the Booster to produce this input set.

The lesson of these often complicated exercises appears to be that the more successful matching computations produce a region of low and gently sloping dispersion in the QH7A - QV7B region. This allows these two quads to be used to tune the relative $X$ and 
Y phase advances needed at the AGS end of the line without markedly affecting the final dispersion. As a result, the matching is credible, with rather broad minima in each of the fitted magnet current parameters, all at about the same central penalty value. Final dispersion is adjusted mainly by means of the QH4 - QV5 - QH6 quads, which balance dispersion against amplitudes in the bend region. After some further tuning, the sensitivity summaries from the matching runs indicated that the two additional quads in the Q10 - Q12 region effectively stabilize the line, an advantage somewhat hidden in the normal mechanics of operating these fitting programs.

This fitted parameter summary data given in Table 4 is intended to show the range of a fitted variable over which a zero penalty function results. With the window form of constraints applied here, a penalty is incurred only when a trial result lies outside of the error assigned to a constrained quantity. When used under the new Revised parameter option, the simplex command explores in more detail the parameter space in the neighborhood of a zero penalty fit, producing two tabulations. For the first, it iterates each variable to either side of its value when the zero penalty result is achieved, the Value field. The values where some constraint is first violated are recorded in the Vlow and Vhigh columns, and the difference is the Spread. Here the scanning for these edges is taken through the third decimal place, and the matched value restored for each parameter in turn. For the second tabulation, the Vlow and Vhigh borders are found, and the average of these two recorded as the central Value. Each such central value is then used for all succeeding parameter evaluations of the second part of the table. The column of central values is very close to the optimum match if the region of the minimum is well behaved in all of the variables. These central values are returned as the final fit on the Match Summary listing. Either of the spread fields is an indirect measure of the sensitivity to the variable, if the penalty function minimizes to about the same value for all variables. A wide spread is better than a narrow one. In the Table 4 listing only the QH4 current appears suspect; in fact changing it only barely grazes an amplitude limit nearby, which is harmless in this example.

As a cautionary note, the window form of constraint, which can lead to a zero penalty, does not necessarily center the final fitted results within the tolerances, or margins of error, that define the windows. Instead this centering has to be achieved by repeating the matching calculations with successively smaller tolerances, which in turn will usually reduce the parameter "spreads" reported on the zero penalty summary tables. In principle the program can be asked to iterate towards smaller constraint tolerances, but so far it has proved more useful to follow the behavior of the fitting, and make adjustments to the constraints as indicated.

Another way to evaluate the sensitivities is to display the amplitude and dispersion functions when the various fitting parameters are iterated over small intervals, using the BNL MAD graphics features. Some Case 3 results are shown in the set of Figures. In Figure 3, the incoming momentum offset from the Booster is varied from -.02 through + .02 . In Figure 4 , the first seven quad currents are varied in turn from -2 through +2 amperes of their fitted values. Quad current errors appear to have very little effect. Similar behavior is obtained from the remaining quads, not shown here. Quad QH4 is the most finicky of the lot. In Figure 5, sensitivities of the beam functions to changes in incoming amplitudes and dispersion are shown, for betas of $-2 \mathrm{~m}$ through $+2 \mathrm{~m}$ about the Set 3 initial values. Similar curves are available for the incoming alphas. While there is 
some amplification of these functions by the line, there is also now the capacity to retune the line accordingly. In Figure 6, the sensitivities to incoming Disp Px are given for the range of -.2 through +.2 .

These results are far superior to the present BTA performance, and appear to be aided by adding both pairs of quads. Undoubtedly compulsive optimization can do somewhat better, but there do seem to be attractive possibilities for improving this line. The possible issue of two versus four new quads is not pressed further here. The final dispersion here depends somewhat too much on QH4 because of the jammed together bend region optics, which have not been modified in any serious way in these studies. We do note that Fermilab uses 21 quads for their corresponding transfer line, with similar emphasis on clamping down amplitudes[3]. The momentum acceptance of Figure 3 may suggest that sextupoles might be considered.

\section{BTA Lattice Changes}

The particular set of BTA lattice changes for the Case 3 configuration is summarized in Table 5. This case is presented merely as a tentative, feasible plan, based mainly on effective transport by quads and bends, and is not a detailed design. The main guidelines are to introduce four additional quads, using the larger of the existing drift spaces, and centering $\mathrm{V}$ quads between $\mathrm{H}$ quads throughout. Present bend, monitor, and foil positions have not been disturbed. One additional quad pair is inserted in the larger drift between QV7 and QH8 in the present shield wall. A second quad pair is inserted in the QH10 - QV13 region, quad spacing is balanced, and QV11 and QH12 polarities are reversed. These emplacements use existing openings, and are not likely to be unique or critical. Some monitors should undoubtedly be shifted to correspond to the revised optics, and the foil centered. All that is suggested here is that these guidelines lead fairly easily to a decently behaved beam line. A tracking run through this configuration is appended.

\section{Fitting Techniques}

These experiences have produced a number of improvements in the fitting programs and some appreciation of the problems in guiding these programs through conflicting multiparameter searches. The BNL MAD Program can now be asked to tabulate the behavior of the fitting penalty functions in the region of the minima in each variable. While the conventional MAD Match listings point towards troubled fits by means of the retry traces and error columns, they do not help very much with situations where the constraints may conflict so that the apparent minima do not coincide. Inconclusive fitting can be aggravated by large numbers of free parameters, and penalty landscapes with numerous deep minima. As already discussed, the case of too few variables for the number of constraints is not very well diagnosed by the usual techniques, and the perhaps cavalier use of amplitude constraints in particular can bring on this condition. The hints for the BTA came from the apparent sensitivity to initial values of the free parameters. The initial value space was then explored using an optional simplex feature that randomizes initial conditions, searches until an obviously unproductive hole is recognized, and then restarts with a new initial set. A summary line of the starting and ending penalty values is printed for each reset; reviewing this information gives a crude picture of the landscape. If there are too many constraints, these restarts will not return to the same 
hole.

It is helpful to explore the effect of the constraints by changing constraint values, such as the clamps on amplitudes. Often adjusting the constraints can be a good way to steer the searches, with a lot of give and take leading to better behaved solutions. When using the window form of constraints, it is usually best to start with broad tolerances, and then reduce them selectively when a promising region is found. Adjusting the relative weighting also can influence the parameter space searches, with quite different numerical effects than adjusting the constrained values. If a match is troubled, usually some common sense based on the relative constraint behaviors should result in more realistic and better behaved matching conditions.

While the simplex method usually samples a large region of the parameter space for a properly stated problem, it can often be helped along when the apparent convergence does not balance all of the constraints as well as it might. For example, a calculation might be stuck fine tuning for some small amplitude gains, while the final dispersion is way off. Here, relaxing an amplitude constraint might lead to a better overall matching. The simplex method can sometimes miss a promising region or a critical parameter influence because the search grid is too large. Also when the search resets itself internally, it repeats with larger initial step sizes. One of the methods we used was to reset the simplex with starting values from a previous run that seemed promising, but had eventually churned without much improvement beyond the early iterations. These starting steps from the FVary commands can be reduced by a common factor entered optionally on the Simplex command, avoiding a lot of editing. This kind of guidance of step size proved very helpful in the eventual solution presented above.

A randomizing feature has been introduced that throws some noise into the later stages of the simplex reduction. Hopefully this helps with a landscape with a broad valley containing local minima, and should serve to reduce some lingering dependences on the order of free variables in the fitting lists. While helpful, the effects have not been fully evaluated in this application.

\section{Comments}

These notes have considered only rather modest BTA changes, ones that leave the Booster connection alone. Partly because the kinds of suggested Booster operating changes have drawn far less hostility than expected, this path has been followed here in studying dispersion issues. Another course, such as moving the Booster connection back a few magnets to a negative dispersion region, has not been reviewed here. This would have obvious advantages for balancing the bend dispersion in the BTA. Similarly a third course would be to split the present two BTA bends into a more gentle curve of four bends, with appropriate clamping of the local dispersion among the bend pairs.

Looked at more critically, the present operational settings depend upon a Match setup that is dominated by perceived AGS dispersion input criteria. This particular class of match permits a small relative adjustment among $\mathrm{X}$ and $\mathrm{Y}$ phases and dispersion by accepting large amplitudes in passage. These paper settings are then trimmed slightly in practice by a few amps to balance BTA aperture losses against AGS acceptance losses. Via some perverse logic, some needle is threaded to balance such losses to some large remnant. This arrangement is particularly sensitive to momentum shifts. The real cost of these losses is substantial, borrowed capital that will have to be repaid some day, but this 
issue is not argued further here. Whatever the usefulness of this style to AGS experiments apparently tolerant of beam quality, using the BTA as a collimator is not likely to offer reproducible, low emittance beams to RHIC for long lasting fills. Hopefully the present studies which look at a few of the possibly easy cases will encourage better BTA designs with adequate tuning among phases and dispersion while limiting emittances.

On the matter of matching techniques, the search algorithms in use here date from the early 1960's, and have not been substantially changed since their introduction. While this is not necessarily bad, somewhat newer approaches to the simplex reduction deal with the reduction of the simplex volume, rather than trying to shorten the longest edge, and thereby avoid some of the quirks due to step size and order of iteration among the innards.

\section{Acknowledgements}

These studies have benefited from numerous discussions among AGS colleagues, and in particular E. Bleser, M. Blaskiewicz, C. Gardner, J. W. Glenn, T. Roser, A. Soukas, and M. Syphers. S. Mane has been particularly helpful. Mistakes are solely from the writer.

\section{References}

1. J. Niederer, BNL MAD Program Notes: BTA Lattice Matching. AGS/AD Tech Note 451. Internal Report.

2. C. Gardner. AGS Internal Report. (In preparation)

3. M. Syphers.

\section{Documents}

Unix Typesetter Format - troff / psroff Files.

\section{Host}

This Report

BTA Lattice Matching

FMatch

Runtwiss

\section{rapt.ags.bnl.gov}

/usr/disc2/jn/Docum+/BTA.notes1 /usr/disc2/jn/Docum+/Match.notes /usr/disc2/jn/Docum+/Fmatch.man /usr/disc2/jn/Docum+/Runtwiss.man

To Print from rapt: (To Room 218 AGS 2nd Floor) alias it. 'cat * psroff -t -ms > ppp; lp ppp'

it. Manual Name 
Table 1. Typical BTA Entrance Parameters

\begin{tabular}{|l|c|c|c|}
\hline Parameter & Original Value & BTA Fitted Value & Booster Value \\
\hline \hline Set & 1 & 2 & 3 \\
\hline Alfax & 1.85 & 2.99 & 3.38 \\
\hline Betax & 13.10 & 18.57 & 22.25 \\
\hline Alfay & -.60 & -.25 & -.54 \\
\hline Betay & 3.87 & 8.43 & 3.40 \\
\hline Dx & 2.85 & .178 & 0. \\
\hline Dpx & -.427 & .463 & 0. \\
\hline
\end{tabular}

Table 2. AGS Entrance Parameter Sets

\begin{tabular}{|l|c|c|}
\hline Parameter & Value & Error \\
\hline \hline Alfax & -.63 & .05 \\
\hline Betax & 10.44 & .5 \\
\hline Alfay & 1.20 & .05 \\
\hline Betay & 20.27 & .5 \\
\hline Dx & -.4085 & .05 \\
\hline Dpx & -.1845 & .05 \\
\hline
\end{tabular}

Table 3. Qualitative Results of Matching

\begin{tabular}{|l|c|c|c|c|}
\hline \multicolumn{1}{|c|}{ Changes } & BTA Case & Input Set 1 & Input Set 2 & Input Set 3 \\
\hline \hline Original & 1 & Bad & Very Good & Bad \\
\hline$\# 1+$ QH7A + QH7B & 2 & Bad & - & Good \\
\hline$\# 2+$ QV10A + QH11B & 3 & Maybe & - & Very Good \\
\hline$\# 1+$ QV10A + QH11B & 4 & Bad & - & Bad \\
\hline
\end{tabular}


Table 4. Region of Fit for BTA Case 3. Aug 5.96

\begin{tabular}{clccccc}
\multicolumn{2}{c}{ Boundaries of } & Zero Penalty & Simplex Fit - Original Fitted Values. \\
Var Var Name & Value & V Low & V High & Spread & Last DV \\
1 & IQV1 & 359.14 & 358.60 & 360.13 & 1.524 & .001 \\
2 & IQH2 & 417.38 & 417.13 & 420.67 & 3.539 & .001 \\
3 & IQV3 & 540.51 & 539.68 & 542.85 & 3.169 & .001 \\
4 & IQH4 & 574.15 & 574.03 & 574.21 & .1840 & .001 \\
5 & IQV5 & 590.17 & 582.50 & 591.19 & 8.694 & .001 \\
6 & IQH6 & 722.67 & 722.57 & 723.07 & .5020 & .001 \\
7 & IQV7 & 438.93 & 437.93 & 439.23 & 1.304 & .001 \\
8 & IQH7A & 231.33 & 230.64 & 232.99 & 2.346 & .001 \\
9 & IQV7B & 747.16 & 743.09 & 761.51 & 18.41 & .001 \\
10 & IQH8 & 556.71 & 555.97 & 563.69 & 7.719 & .001 \\
11 & IQV9 & 701.11 & 699.03 & 701.81 & 2.778 & .001 \\
12 & IQH10 & 426.59 & 426.45 & 432.47 & 6.018 & .001 \\
13 & IQV10A & 266.88 & 263.67 & 267.08 & 3.408 & .001 \\
14 & IQH11A & 373.32 & 373.19 & 374.91 & 1.718 & .001 \\
15 & IQV12A & 578.22 & 575.37 & 578.68 & 3.301 & .001 \\
16 & IQH12B & 353.40 & 353.09 & 354.51 & 1.426 & .001 \\
17 & IQV13 & 679.92 & 677.19 & 681.34 & 4.143 & .001 \\
18 & IQH14 & 569.37 & 568.36 & 570.69 & 2.322 & .001 \\
19 & IQV15 & 166.39 & 165.45 & 169.40 & 3.955 & .001
\end{tabular}

Boundaries of Zero Penalty Simplex Fit - Central Values.

\begin{tabular}{|c|c|c|c|c|c|c|}
\hline Var & Var Name & Value & V Low & V High & Spread & Last DV \\
\hline 1 & IQVI & 359.36 & 358.60 & 360.13 & 1.524 & .001 \\
\hline 2 & IQH2 & 418.96 & 417.17 & 420.74 & 3.570 & .001 \\
\hline 3 & IQV3 & 546.23 & 539.04 & 553.41 & 14.360 & .001 \\
\hline 4 & IQH4 & 574.17 & 573.62 & 574.72 & 1.095 & .001 \\
\hline 5 & IQV5 & 589.86 & 584.77 & 594.95 & 10.180 & .001 \\
\hline 6 & IQ̃H6 & 723.18 & 721.77 & 724.60 & 2.833 & .001 \\
\hline 7 & IQV7 & 440.54 & 437.89 & 443.20 & 5.310 & .001 \\
\hline 8 & IQ̈H7A & 229.33 & 224.86 & 233.81 & 8.949 & .001 \\
\hline 9 & IQ̃V7B & 744.62 & 735.23 & 754.01 & 18.780 & .001 \\
\hline 10 & IQHH8 & 556.73 & 552.35 & 561.12 & 8.767 & .001 \\
\hline 11 & IQV9 & 701.45 & 700.04 & 702.86 & 2.827 & .001 \\
\hline 12 & IQHI 0 & 428.24 & 425.83 & 430.64 & 4.810 & .001 \\
\hline 13 & IQV10A & 265.33 & 260.32 & 270.35 & 10.030 & .001 \\
\hline 14 & $I Q H \perp I A$ & 374.40 & 370.49 & 378.32 & 7.821 & .001 \\
\hline 15 & IQVI2A & 576.51 & 573.66 & 579.35 & 5.689 & .001 \\
\hline 16 & IQH12B & 353.73 & 347.88 & 359.58 & 11.700 & .001 \\
\hline 17 & IQVI 3 & 677.41 & 669.06 & 685.75 & 16.680 & .001 \\
\hline 18 & IQH]14 & 569.99 & 566.58 & 573.39 & 6.813 & .001 \\
\hline 19 & IQVV15 & 167.28 & 165.38 & 169.17 & 3.797 & .001 \\
\hline
\end{tabular}


Table 5. Survey of Case 3 BTA Lattice Changes

\begin{tabular}{|l|c|l|c|l|}
\hline Original & $\begin{array}{c}\text { Value } \\
(\mathrm{m})\end{array}$ & Revised & $\begin{array}{c}\text { Value } \\
(\mathrm{m})\end{array}$ & \multicolumn{1}{|c|}{ Note } \\
\hline \hline DR019 & .32570 & DR019A & .59020 & Center QV3 \\
\hline DRQ3 & .37060 & DRQ3A & .10610 & " \\
\hline DRD2 & .47335 & DRD2A & .72915 & Center QV5 \\
\hline DR046 & .86660 & DR046A & .61080 & " \\
\hline DR060 & .40615 & DR060A & 1.00940 & Balance Spacing \\
\hline & & & & \\
DRQ7Y & 4.25075 & DRQ7AA & 1.95075 & Shorten \\
\hline & & QH7A & .49850 & New Quad \\
\hline DRQ7Z & 4.25075 & DRQ7B & 2.50450 & Shorten \\
\hline & & QV7B & .49850 & New Quad \\
\hline & & DRQ7C & 2.50000 & \\
\hline DRQ10 & 5.03015 & DRQ10A & 2.81050 & Shorten \\
\hline & & QV10A & .49850 & New Quad \\
\hline & & DRQ10B & 1.72130 & Quad Space \\
\hline QV11 & .49850 & QH11A & .49850 & Reverse Quad \\
\hline DRQ11 & 3.01295 & DRQ11A & 1.61295 & Shorten \\
\hline QH12 & .49850 & QV12A & .49850 & Reverse Quad \\
\hline DRQ12 & 3.78025 & DRQ12A & .27803 & Shorten \\
\hline & & QH12B & .49850 & New Quad \\
\hline & & DRQ12B & 1.90150 & Balance Spacing \\
\hline
\end{tabular}




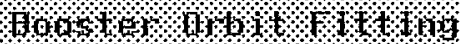

FTW. $5[$ QX]

4.7993

FTW. 5[ QY]

4. 8020

PDELTH

$$
\text { . DO }
$$

FTW. 5[ BETXMAS

13.569

FTW.5[ DKMAX]

2.9546

FTW. 5[ BETYMA?

13.670

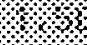

\%

\%

\%

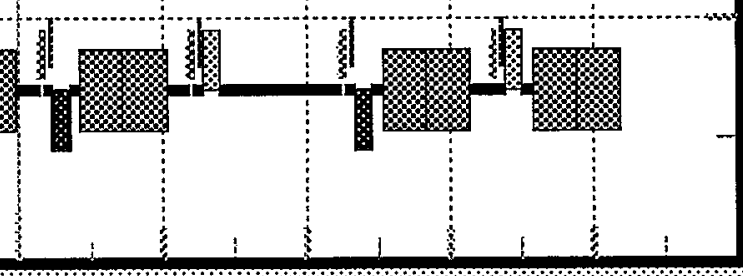

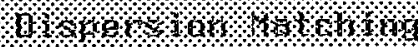

101010101

m

10101\%

101010

Figure 1. Normal dispersion Dx and DPx in the F region of the Booster. 


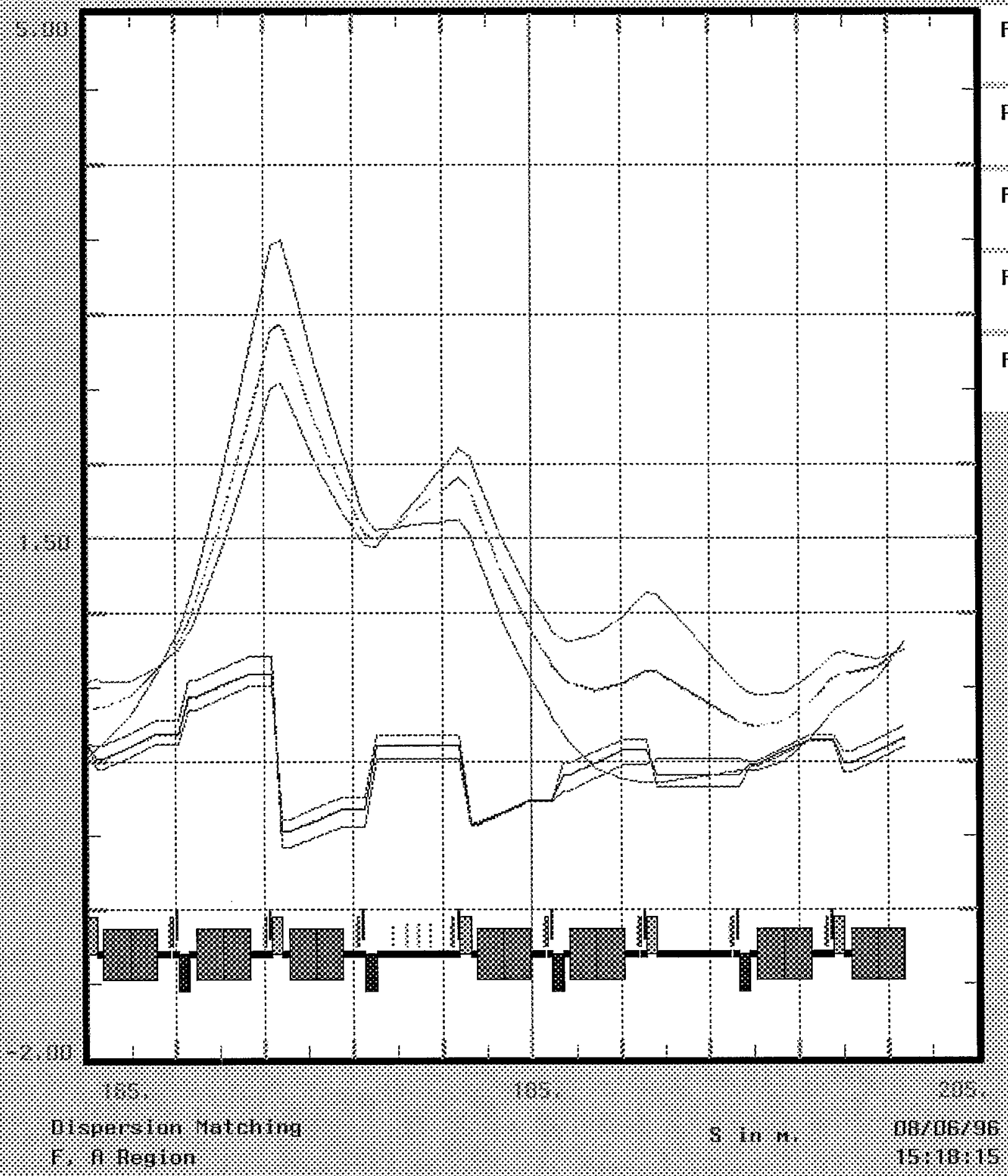

FTW. 5[ QY]

4, 7851

PDELTH

.00

FTW. 5[ BETXMAS 27.744

FTW. 5[ DXMAX]

5.4485

FTW.5[ BETYMA?

15.167 
o

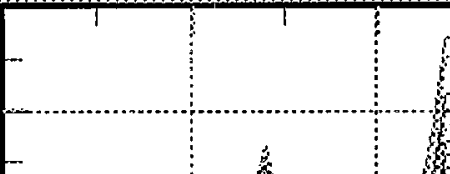

3010



की
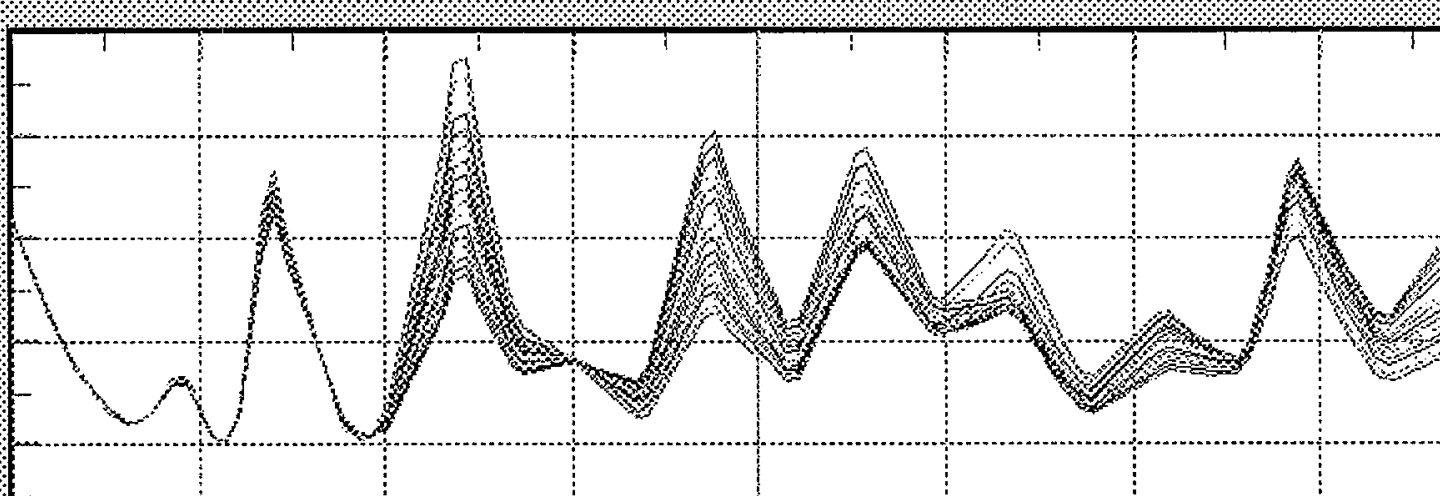

101

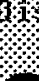
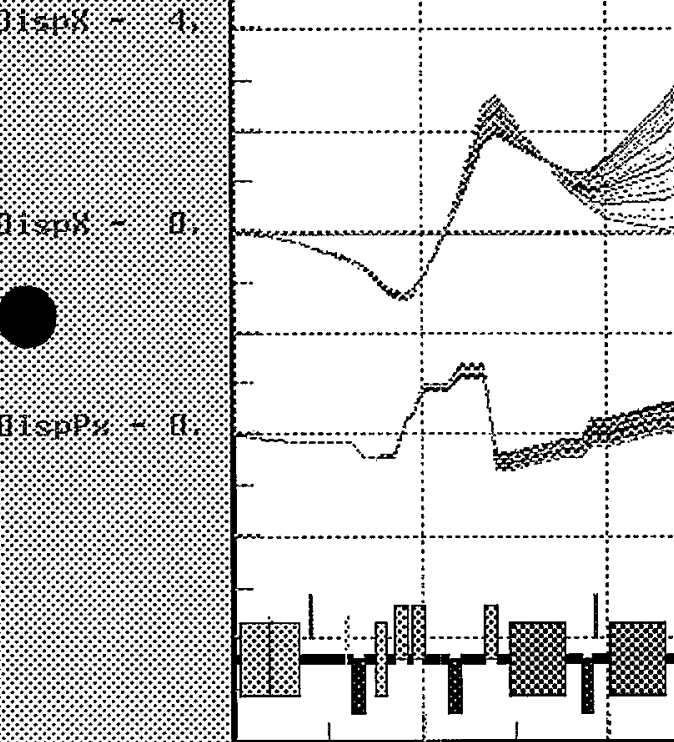

\%

Figure 3. Momentum dependence of revised BTA tracking. 


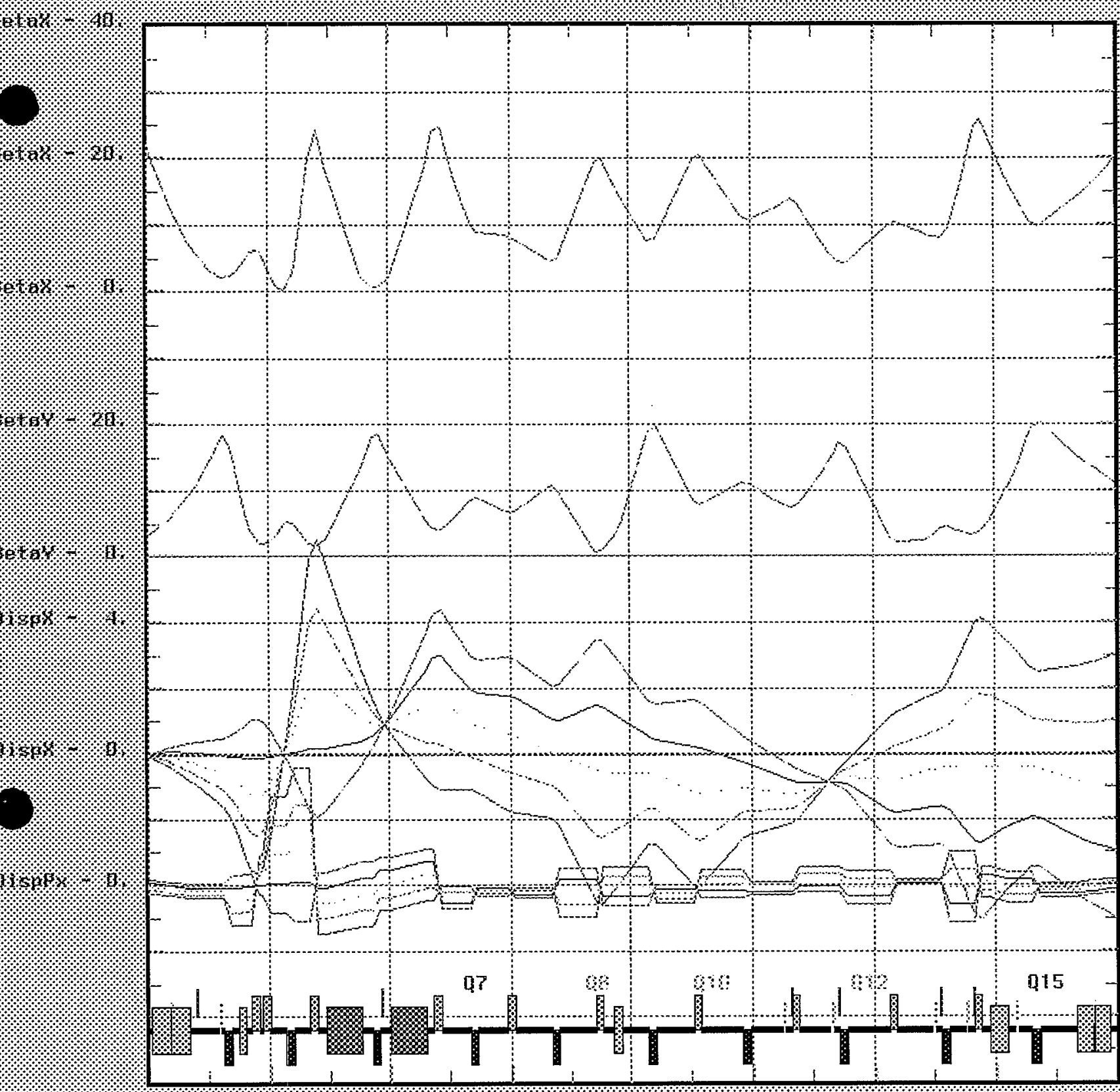

Figure 6. Dependence of revised BTA on initial Disp Px. 
LINEAR LATTICE PARAMETERS FOR BEAM LINE: "BTA 3 ", RANGE = "\#S / \#E"

\section{ELEMENT SEQUENCE}

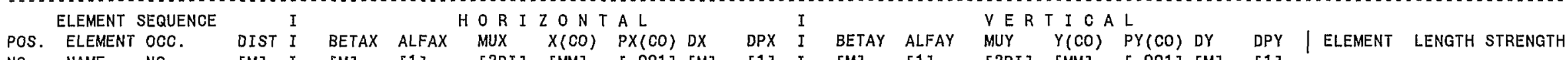

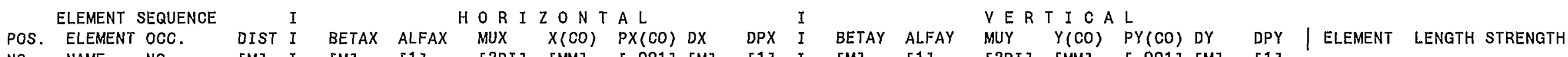
$[2 \mathrm{PI}][\mathrm{MM}] \quad[.001][\mathrm{M}] \quad[1]$ I [2PI] $[\mathrm{MM}][.001][\mathrm{M}][1]$

\begin{tabular}{|c|c|c|c|c|c|c|c|c|c|c|c|c|c|c|c|c|c|c|c|}
\hline BEGIN & BTA_3 & 1 & 0.000 & 22.250 & 3.380 & 0.000 & 0.000 & 0.000 & $0.000 \quad 0.000$ & 3.400 & -0.540 & 0.000 & 0.000 & 0.000 & 0.000 & 0.000 & & & \\
\hline BEGIN & $\mathrm{LO}^{-}$ & 1 & 0.000 & 22.250 & 3.380 & 0.000 & 0.000 & 0.000 & $0.000 \quad 0.000$ & 3.400 & -0.540 & 0.000 & 0.000 & 0.000 & 0.000 & 0.000 & & & \\
\hline 1 & MQHF6 & 1 & 0.000 & 22.250 & 3.380 & 0.000 & 0.000 & 0.000 & $0.000 \quad 0.000$ & 3.400 & -0.540 & 0.000 & 0.000 & 0.000 & 0.000 & 0.000 & MARKER & 0.00000 & 0.0000 \\
\hline 2 & DRQF6 & 1 & 0.236 & 20.685 & 3.248 & 0.002 & 0.000 & 0.000 & 0.0000 .000 & 3.676 & -0.630 & 0.011 & 0.000 & 0.000 & 0.000 & 0.000 & DRIFT & 0.23610 & 0.0000 \\
\hline 3 & DHF6A & 1 & 1.486 & 13.358 & 2.549 & 0.014 & 0.000 & 0.000 & $-0.049-0.078$ & 5.844 & -1.081 & 0.054 & 0.000 & 0.000 & 0.000 & 0.000 & SBEND & 1.25000 & -0.0715 \\
\hline 4 & DRF6A1 & 1 & 1.506 & 13.257 & 2.538 & 0.014 & 0.000 & 0.000 & $-0.051-0.078$ & 5.887 & -1.088 & 0.055 & 0.000 & 0.000 & 0.000 & 0.000 & DRIFT & 0.01975 & 0.0000 \\
\hline 5 & DHF6T & 1 & 1.506 & 13.257 & 2.538 & 0.014 & 0.000 & 0.000 & $-0.051-0.078$ & 5.887 & -1.088 & 0.055 & 0.000 & 0.000 & 0.000 & 0.000 & HKICK & 0.00000 & 0.0000 \\
\hline 6 & DRF6A2 & 1 & 1.526 & 13.157 & 2.527 & 0.014 & 0.000 & 0.000 & $-0.052-0.078$ & 5.930 & -1.095 & 0.055 & 0.000 & 0.000 & 0.000 & 0.000 & DRIFT & 0.01975 & 0.0000 \\
\hline 7 & DHF6B & 1 & 2.853 & 7.447 & 1.783 & 0.036 & 0.000 & 0.000 & $-0.199-0.144$ & 9.460 & -1.563 & 0.084 & 0.000 & 0.000 & 0.000 & 0.000 & SBEND & 1.32690 & -0.0594 \\
\hline 8 & DRF6B & 1 & 3.259 & 6.091 & 1.555 & 0.045 & 0.000 & 0.000 & $-0.258-0.144$ & 10.791 & -1.711 & 0.090 & 0.000 & 0.000 & 0.000 & 0.000 & DRIFT & 0.40640 & 0.0000 \\
\hline END & LO & 1 & 3.259 & 6.091 & 1.555 & 0.045 & 0.000 & 0.000 & $-0.258-0.144$ & 10.791 & -1.711 & 0.090 & 0.000 & 0.000 & 0.000 & 0.000 & & & \\
\hline BEGIN & L1 & 1 & 3.259 & 6.091 & 1.555 & 0.045 & 0.000 & 0.000 & $-0.258-0.144$ & 10.791 & -1.711 & 0.090 & 0.000 & 0.000 & 0.000 & 0.000 & & & \\
\hline 9 & PUEH001 & 1 & 3.259 & 6.091 & 1.555 & 0.045 & 0.000 & 0.000 & $-0.258-0.144$ & 10.791 & -1.711 & 0.090 & 0.000 & 0.000 & 0.000 & 0.000 & MONITOR & 0.00000 & 0.0000 \\
\hline 10 & DR001 & 1 & 4.344 & 3.378 & 0.946 & 0.084 & 0.000 & 0.000 & $-0.413-0.144$ & 14.933 & -2.106 & 0.104 & 0.000 & 0.000 & 0.000 & 0.000 & DRIFT & 1.08490 & 0.0000 \\
\hline 11 & MW006 & 1 & 4.344 & 3.378 & 0.946 & 0.084 & 0.000 & 0.000 & $-0.413-0.144$ & 14.933 & -2.106 & 0.104 & 0.000 & 0.000 & 0.000 & 0.000 & MARKER & 0.00000 & 0.0000 \\
\hline 12 & DRDO6 & 1 & 4.776 & 2.664 & 0.703 & 0.107 & 0.000 & 0.000 & $-0.475-0.144$ & 16.823 & -2.264 & 0.108 & 0.000 & 0.000 & 0.000 & 0.000 & DRIFT & 0.43260 & 0.0000 \\
\hline 13 & DV007 & 1 & 5.005 & 2.372 & 0.575 & 0.121 & 0.000 & 0.000 & $-0.508-0.144$ & 17.877 & -2.347 & 0.110 & 0.000 & 0.000 & 0.000 & 0.000 & VKICK & 0.22860 & 0.0000 \\
\hline 14 & DR007 & 1 & 5.166 & 2.202 & 0.485 & 0.132 & 0.000 & 0.000 & $-0.531-0.144$ & 18.640 & -2.405 & 0.111 & 0.000 & 0.000 & 0.000 & 0.000 & DRIFT & 0.16060 & 0.0000 \\
\hline 15 & QV1 & 1 & 5.724 & 2.413 & -0.898 & 0.173 & 0.000 & 0.000 & $-0.691-0.439$ & 16.263 & 6.259 & 0.116 & 0.000 & 0.000 & 0.000 & 0.000 & QUADRUPO & 0.55880 & -0.8862 \\
\hline 16 & DRQ1 & 1 & 6.128 & 3.260 & -1.200 & 0.196 & 0.000 & 0.000 & $-0.868-0.439$ & 11.618 & 5.263 & 0.121 & 0.000 & 0.000 & 0.000 & 0.000 & DRIFT & 0315 & 0.0000 \\
\hline 17 & DH1 & 1 & 6.661 & 4.752 & -1.600 & 0.218 & 0.000 & 0.000 & $-1.091-0.399$ & 6.695 & 3.961 & 0.131 & 0.000 & 0.000 & 0.000 & 0.000 & RBEND & 0.53330 & 0.0364 \\
\hline END & L1 & 1 & 6.661 & 4.752 & -1.600 & 0.218 & 0.000 & 0.000 & $-1.091-0.399$ & 6.695 & 3.961 & 0.131 & 0.000 & 0.000 & 0.000 & 0.000 & & & \\
\hline BEGIN & L2A & 1 & 6.661 & 4.752 & -1.600 & 0.218 & 0.000 & 0.000 & $-1.091-0.399$ & 6.695 & 3.961 & 0.131 & 0.000 & 0.000 & 0.000 & 0.000 & & & \\
\hline 18 & DRD1 & 1 & 7.040 & 6.074 & -1.884 & 0.229 & 0.000 & 0.000 & $-1.243-0.399$ & 4.048 & 3.016 & 0.142 & 0.000 & 0.000 & 0.000 & 0.000 & DRIFT & 0.37935 & 0.0000 \\
\hline 19 & QH2A & 1 & 7.599 & 6.209 & 1.668 & 0.243 & 0.000 & 0.000 & $\begin{array}{lll}-1.259 & 0.342\end{array}$ & 2.222 & 0.595 & 0.174 & 0.000 & 0.000 & 0.000 & 0.000 & QUADRUPO & 880 & 0318 \\
\hline 20 & DRQ2A & 1 & 7.715 & 5.831 & 1.597 & 0.246 & 0.000 & 0.000 & $\begin{array}{lll}-1.220 & 0.342\end{array}$ & 2.093 & 0.525 & 0.182 & 0.000 & 0.000 & 0.000 & 0.000 & DRIFT & 1580 & 0.0000 \\
\hline 21 & QH2B & 1 & 8.274 & 2.890 & 3.087 & 0.266 & 0.000 & 0.000 & $\begin{array}{lll}-0.847 & 0.954\end{array}$ & 2.333 & -1.001 & 0.225 & 0.000 & 0.000 & 0.000 & 0.000 & QUADRUPO & 0.55880 & 1.0318 \\
\hline 22 & $D R Q 2 B$ & 1 & 8.734 & 0.821 & 1.412 & 0.315 & 0.000 & 0.000 & $-0.409 \quad 0.954$ & 3.435 & -1.395 & 0.251 & 0.000 & 0.000 & 0.000 & 0.000 & DRIFT & 0.45990 & 0.0000 \\
\hline 23 & XF019 & 1 & 8.734 & 0.821 & 1.412 & 0.315 & 0.000 & 0.000 & $\begin{array}{lll}-0.409 & 0.954\end{array}$ & 3.435 & -1.395 & 0.2 & 0.000 & 000 & 0.0 & 0.000 & MARKER & 000 & 0000 \\
\hline 24 & DR019A & 1 & 9.324 & 0.424 & -0.739 & 0.568 & 0.000 & 0.000 & $0.155 \quad 0.954$ & 5.381 & -1.901 & 0.273 & 0.000 & 0.000 & 0.000 & 0.000 & DRIFT & 1020 & 0.0000 \\
\hline 25 & QV3 & 1 & 9.883 & 3.003 & -4.496 & 0.657 & 0.000 & 0.000 & 0.7591 .282 & 5.260 & 2.087 & 0.289 & 0.000 & 0.000 & 0.000 & 0.000 & QUADRUPO & 5880 & -1.3271 \\
\hline 26 & DRQ3A & 1 & 9.989 & 4.036 & -5.246 & 0.662 & 0.000 & 0.000 & $0.895 \quad 1.282$ & & 1.979 & 0.292 & 0.000 & 0.000 & & 100 & DRIFT & 0610 & 0.0000 \\
\hline 27 & FOILO24 & 1 & 9.989 & 4.036 & -5.246 & 0.662 & 0.000 & 0.000 & $0.895 \quad 1.282$ & 4.829 & 1.979 & 0.292 & 0.000 & 0.000 & 0.000 & 0.000 & MARKER & 0.00000 & 0.0000 \\
\hline END & L2A & 1 & 9.989 & 4.036 & -5.246 & 0.662 & 0.000 & 0.000 & 0.8951 .282 & 4.829 & 1.979 & 0.292 & 0.000 & 0.000 & 0.000 & 0.000 & & & \\
\hline BEGIN & L2B & 1 & 9.989 & 4.036 & -5.246 & 0.662 & 0.000 & 0.000 & $0.895 \quad 1.282$ & 4.829 & 1.979 & 0.292 & 0.000 & 0.000 & 0.000 & 0.000 & & & \\
\hline 28 & DRO24 & 1 & & & 11.915 & 0.6 & 0.000 & 0.000 & 151.282 & 2.000 & 1.018 & & 000 & 000 & 000 & 0.000 & $D R$ & & 0.0000 \\
\hline 29 & QH4 & 1 & .491 & 24.244 & 5.823 & 0.682 & 0.000 & 0.000 & $2.325-0.522$ & 1.879 & -0.771 & 0.391 & 0.000 & 0.000 & 0.000 & 0.000 & QUADRUP & 5880 & 1.4036 \\
\hline 30 & DRQ4 & 1 & 11.980 & 18.902 & 5.120 & 0.686 & 0.000 & 0.000 & $2.071-0.522$ & 2.834 & -1.185 & 0.425 & 0.000 & 0.000 & 0.000 & 0.000 & DRIFT & 0.48810 & 0.0000 \\
\hline 31 & $\mathrm{DH} 2$ & 1 & 14.400 & 2.368 & 1.645 & 0.744 & 0.000 & 0.000 & $1.141-0.247$ & 13.364 & -3.104 & 0.489 & 0.000 & 0.000 & 0.000 & 0.000 & SBEND & 2.42000 & 0.2757 \\
\hline END & L2B & 1 & & 2.368 & 1.645 & & 0.000 & & $1.141-0.247$ & & & & & 0.000 & 0.000 & 0.000 & & & \\
\hline BEGIN & L3 & 1 & 14.400 & 2.368 & 1.645 & 0.744 & 0.000 & 0.000 & $1.141-0.247$ & 13.364 & -3.104 & 0.489 & 0.000 & 0.000 & 0.000 & 0.000 & & & \\
\hline 32 & DRD2A & 1 & 15.129 & 0.801 & 0.504 & 0.832 & 0.000 & 0.000 & $0.961-0.247$ & 18.313 & -3.684 & 0.497 & 0.000 & 0.000 & 0.000 & 0.000 & DRIFT & 0.72915 & 0.0000 \\
\hline 33 & QV5 & 1 & 15.627 & 0.797 & -0.494 & 0.943 & 0.000 & 0.000 & $0.918 \quad 0.072$ & 18.792 & 2.778 & 0.501 & 0.000 & 0.000 & 0.000 & 0.000 & QUADRUPO & 0.49850 & -0.6921 \\
\hline 34 & DRQ5 & 1 & 15.746 & 0.936 & -0.679 & 0.965 & 0.000 & 0.000 & $0.926 \quad 0.072$ & 18.141 & 2.723 & & & 0.000 & 0.000 & 0.000 & DRIFT & 0.11835 & 0.0000 \\
\hline 35 & PUEV046 & 1 & 15.746 & 0.936 & -0.679 & 0.965 & 0.000 & 0.000 & $\begin{array}{lll}0.926 & 0.072\end{array}$ & 18.141 & 2.723 & 0.502 & 0.000 & 0.000 & 0.000 & 0.000 & MONITOR & 0.00000 & 0.0000 \\
\hline 36 & DR046A & 1 & 16.356 & 2.348 & -1.633 & 1.033 & 0.000 & 0.000 & 0.9710 .072 & 14.987 & 2.440 & 0.508 & 0.000 & 0.000 & 0.000 & 0.000 & DRIFT & 0.61080 & 0.0000 \\
\hline 37 & DH3 & 1 & 18.776 & 18.809 & & 1.091 & 0.000 & 0.000 & $1.485 \quad 0.354$ & & 1.370 & 0.550 & 0.000 & 0.000 & 0.000 & 0.000 & SBEND & 2.42000 & 0.2757 \\
\hline END & L3 & 1 & 18.776 & 18.809 & -5.102 & 1.091 & 0.000 & 0.000 & $1.485 \quad 0.354$ & 5.633 & 1.370 & 0.550 & 0.000 & 0.000 & 0.000 & 0.000 & & & \\
\hline
\end{tabular}


